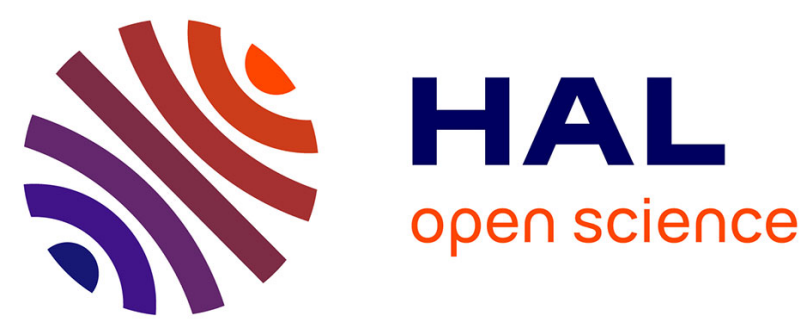

\title{
Potential energy surface and rovibrational bound states of the H2-C3N- van der Waals complex
}

Miguel Lara-Moreno, Thierry Stoecklin, Philippe Halvick

\section{To cite this version:}

Miguel Lara-Moreno, Thierry Stoecklin, Philippe Halvick. Potential energy surface and rovibrational bound states of the H2-C3N- van der Waals complex. Physical Chemistry Chemical Physics, 2019, 21, pp.2929 - 2937. 10.1039/c8cp07727d . hal-03044683

\section{HAL Id: hal-03044683 \\ https://cnrs.hal.science/hal-03044683}

Submitted on 14 Dec 2020

HAL is a multi-disciplinary open access archive for the deposit and dissemination of scientific research documents, whether they are published or not. The documents may come from teaching and research institutions in France or abroad, or from public or private research centers.
L'archive ouverte pluridisciplinaire HAL, est destinée au dépôt et à la diffusion de documents scientifiques de niveau recherche, publiés ou non, émanant des établissements d'enseignement et de recherche français ou étrangers, des laboratoires publics ou privés. 


\title{
Potential energy surface and rovibrational bound states of the $\mathrm{H}_{2}-\mathrm{C}_{3} \mathbf{N}^{-}$van der Waals complex
}

\author{
Miguel Lara-Moreno, ${ }^{1}$ Thierry Stoecklin, ${ }^{1}$ and Philippe Halvick ${ }^{1, a)}$ \\ Université de Bordeaux, ISM, CNRS UMR 5255, 33405, Talence, \\ France
}

(Dated: 11 January 2019)

Since their recent detection in the interstellar medium, anions have raised the question of their possible mechanisms of formation, destruction and excitation. This require the knowledge of their interaction with the most abundant interstellar species. In the present work, a four dimensional rigid rotor model of the potential energy surface is developed for the collision of $\mathrm{C}_{3} \mathrm{~N}^{-}$with $\mathrm{H}_{2}$. Ab initio calculations are performed with explicitly-correlated coupled-cluster theory via CCSD(T)-F12/augcc-pVTZ. Two linear equilibrium structures are found, different by the orientation of $\mathrm{C}_{3} \mathrm{~N}^{-}$. Two more equilibrium structures, symmetrically equivalent, are obtained by the permutation of $\mathrm{H}$ atoms. The vibrational dynamics is mainly controlled by the considerable difference between the two bending frequencies which corresponds to the hindered rotations of $\mathrm{C}_{3} \mathrm{~N}^{-}$and $\mathrm{H}_{2}$. This arises from the potential energy surface which is soft for the rotation of $\mathrm{C}_{3} \mathrm{~N}^{-}$and stiff for rotation of $\mathrm{H}_{2}$, and also from the large difference in mass between both monomers. Although a high barrier of potential prevents the rotation of $\mathrm{H}_{2}$, a significant tunneling effect is observed which causes a splitting in the degenerate energy levels. On the contrary, the rotation of $\mathrm{C}_{3} \mathrm{~N}^{-}$is allowed since the energy of the saddle points is lower than the energy of the bound states, but the wavefunctions remain localized around each linear structures unless a large excitation energy is available.

a)E-mail:philippe.halvick@u-bordeaux.fr 


\section{INTRODUCTION}

Since the late 1900s, interstellar anions have been a controversial topic in the astrochemistry community ${ }^{1-7}$ as they are not only exotic molecules for such harsh environments, but also could contribute to the growing of molecular complexity in the interstellar medium (ISM). A keystone contribution to the field was the detection of the first interstellar anion $\mathrm{C}_{6} \mathrm{H}^{-}$in $2006^{8}$. This discovery opened the gate to careful spectral explorations and subsequent detection of five more anions ${ }^{9-14}$, among them $\mathrm{C}_{3} \mathrm{~N}^{-}$which has a large dipole moment and is remarkably stable since $\mathrm{C}_{3} \mathrm{~N}$ has a large electron affinity ${ }^{15}, 4.3 \mathrm{eV}$.

The anion-to-neutral ratios observed in the ISM are of the order of a percent ${ }^{16}$. The column density of $\mathrm{C}_{3} \mathrm{~N}^{-}$is $0.5 \%$ that of $\mathrm{C}_{3} \mathrm{~N}$ in $\mathrm{IRC}+10216^{14}$. An upper limit of $0.8 \%$

of the latter ratio has been observed in TMC- $1^{14}$. These column densities are derived under the assumption of the local thermodynamic equilibrium (LTE). However, in dilute astrophysical environments, non-LTE excitation effects can be responsible for errors in the determination of column densities. In the cold dense molecular clouds where $\mathrm{C}_{3} \mathrm{~N}^{-}$was detected, the most abundant species is $\mathrm{H}_{2}$, and it is expected to induce rotational excitation of $\mathrm{C}_{3} \mathrm{~N}^{-}$by collision. The knowledge of accurate rotational transition rate coefficients is therefore required for predicting the rotational level populations in non-LTE environments. Such calculations rely on the detailed knowledge of the potential energy surface (PES) of the system formed by the two interacting molecules. In this paper, we report an accurate PES for the $\mathrm{H}_{2}-\mathrm{C}_{3} \mathrm{~N}^{-}$system and investigate the properties of this PES by computing and analyzing the bound states wavefunctions.

\section{POTENTIAL ENERGY SURFACE}

\section{A. Ab initio calculations}

As both $\mathrm{C}_{3} \mathrm{~N}^{-}$and $\mathrm{H}_{2}$ are linear closed shell molecules in their ground state, they are well described by a mono-determinantal electronic wavefunction. This makes the coupledcluster method a reliable approach for calculating the interaction potential between these two molecules. The explicitly-correlated coupled-cluster method with single and double excitation complemented by a perturbative treatment of triple excitations (CCSD(T)-F12) has been shown to provide an accurate description of the interaction between charged and 


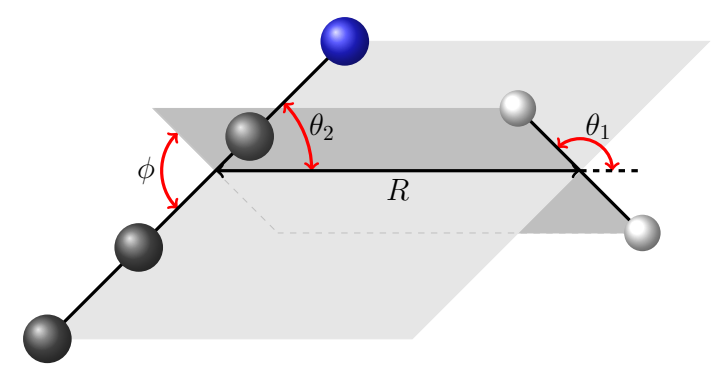

FIG. 1. (color online) Body-fixed Jacobi coordinates used to describe the interaction between $\mathrm{H}_{2}$ and $\mathrm{C}_{3} \mathrm{~N}^{-}$.

neutral molecules ${ }^{17,18}$. Furthermore, the accuracy offered by the F12 approach when using a triple-zeta basis set is comparable to the one of quintuple-zeta quality coupled-cluster calculation without F12. Therefore, this method has been widely used to map multidimensional PES for a reduced computational cost.

Based on the aforementionned features, we have used in the present work the CCSD(T)F12 method with the aug-cc-pVTZ ${ }^{19}$ atomic orbitals basis set, the aug-cc-pVTZ/MP2FIT ${ }^{20}$ density fitting basis set and the cc-pVTZ/JKFIT ${ }^{21}$ resolution of the identity basis set, to calculate the $\mathrm{H}_{2}-\mathrm{C}_{3} \mathrm{~N}^{-}$interaction energies on a $4 \mathrm{D}$ grid of points expressed in the bodyfixed Jacobi coordinates defined in Figure 1. Both monomers are assumed to be linear rigid rotors. Since $\mathrm{C}_{3} \mathrm{~N}^{-}$is a long molecule, the interaction energy is strongly anisotropic for small intermonomer separation. Therefore a large density of ab initio points was necessary to describe properly this region. A total number of 28339 geometries expressed in Jacobi coordinates were calculated with $R$ ranging from 2 to $50 a_{0}, \theta_{1}$ from $0^{\circ}$ to $90^{\circ}, \theta_{2}$ from $0^{\circ}$ to $180^{\circ}$, and $\phi$ from $0^{\circ}$ to $180^{\circ}$.

For all these calculations carried out with the MOLPRO package ${ }^{22}$, the $\mathrm{H}_{2}$ bond length was fixed to its vibrationally averaged value in the rovibrational ground state $r_{\mathrm{HH}}=$ $1.448736 a_{0}$ while the $\mathrm{C}_{3} \mathrm{~N}^{-}$bond lengths were set to their equilibrium values obtained from $\operatorname{CCSD}(\mathrm{T}) /$ aug-cc-pV5Z calculations ${ }^{23}$, namely, $r_{\mathrm{C}_{1} \mathrm{C}_{2}}=2.3653 a_{0}, r_{\mathrm{C}_{2} \mathrm{C}_{3}}=2.5817 a_{0}$, and $r_{\mathrm{C}_{3} \mathrm{~N}}=2.2136 a_{0}$. The basis superposition error (BSSE) was corrected by means of the counterpoise procedure ${ }^{24}$ applied to the rigid monomer case. 


\section{B. Analytical representation of the PES}

The functional form used to fit the ab initio energy points is defined as the sum of the two terms $f_{S R}$ and $f_{L R}$ associated with the short-range and long-range contributions, respectively.

$$
V\left(R, \theta_{1}, \theta_{2}, \phi\right)=S(R) f_{S R}\left(R, \theta_{1}, \theta_{2}, \phi\right)+[1-S(R)] f_{L R}\left(R, \theta_{1}, \theta_{2}, \phi\right)
$$

where the switching function $S(R)$ is defined as:

$$
S(R)=\frac{1}{2}\left[1-\tanh \left(A_{0}\left(R-R_{0}\right)\right)\right]
$$

Each of the two long-range and short-range terms in equation (1) is expressed as a linear combination of products of normalized associated Legendre polynomials $\bar{P}_{l}^{m}$ and cosine functions.

$$
f\left(R, \theta_{1}, \theta_{2}, \phi\right)=\sum_{l_{1} l_{2} m} v_{l_{1}, l_{2}}^{m}(R) \bar{P}_{l_{1}}^{m}\left(\cos \theta_{1}\right) \bar{P}_{l_{2}}^{m}\left(\cos \theta_{2}\right) \cos (m \phi)
$$

In the latter expression, only even values of $l_{1}$ are included as a result of the permutation symmetry of the two $\mathrm{H}$ atoms.

For each point $R_{i}$ of the radial grid, the short-range coefficient $\left[v_{l_{1}, l_{2}}^{m}\left(R_{i}\right)\right]_{S R}$ are obtained through a weighted linear least squares fit of the ab initio data using equation (3). In this step, we used the energy dependent weighting function $w(E)$ in order to obtain the smallest fitting errors in the low energy region of the PES,

$$
w(E)=w_{\min }+\frac{w_{\max }}{2}\left[1-\tanh \left(\alpha\left(E-E_{0}\right)\right)\right]
$$

Additionally, an energy cut-off of $40000 \mathrm{~cm}^{-1}$ was used in order to exclude from the fit the highest energies arising from the monomers repulsion force at short distance of separation and for $\theta_{2}$ close to $0^{\circ}$ or $180^{\circ}$. Then a cubic spline interpolation was performed to obtain the short-range radial functions $\left[v_{l_{1}, l_{2}}^{m}(R)\right]_{S R}$.

The discrete values of the long-range coefficients $\left[v_{l_{1}, l_{2}}^{m}\left(R_{i}\right)\right]_{L R}$ were obtained by applying the same procedure, but using only the ab initio points for $R \geq 15 a_{0}$. Then the long-range radial functions were obtained by a linear least squares fitting of the following expression

$$
\left[v_{l_{1}, l_{2}}^{m}(R)\right]_{L R}=\sum_{k=3}^{8} \frac{t_{k}(\beta R)}{R^{k}} C_{l_{1}, l_{2}, k}^{m}
$$


TABLE I. Stationary points of the $\mathrm{H}_{2}-\mathrm{C}_{3} \mathrm{~N}^{-}$4D PES. The angle $\phi$ is not defined (N/D) for the collinear structures.

\begin{tabular}{lrrrrr}
\hline \hline Point & $R\left(a_{0}\right)$ & $\theta_{1}($ deg. $)$ & $\theta_{2}($ deg. $)$ & $\phi($ deg. $)$ & $D_{e}\left(\mathrm{~cm}^{-1}\right)$ \\
\hline MIN1 & 9.39 & 0 or 180 & 180 & N/D & 769.75 \\
MIN2 & 8.89 & 0 or 180 & 0 & N/D & 561.77 \\
SP1 & 6.40 & 173.40 & 78.01 & 0 & 349.57 \\
SP2 & 5.99 & 97.27 & 81.06 & 0 & 249.54 \\
\hline \hline
\end{tabular}

where $t_{k}$ is the Tang-Toennies damping function:

$$
t_{k}(x)=1-e^{-x} \sum_{i=0}^{k} \frac{x^{i}}{i !}
$$

A total of 243 angular functions, defined by $\left\{l_{1} \in[0,6], l_{2} \in[0,18], m \in[0,4]\right\}$, were needed to reproduce accurately the strong anisotropy of the short-range contribution while only 25 angular functions, defined by $\left\{l_{1} \in[0,2], l_{2} \in[0,6], m \in[0,2]\right\}$, were needed for the accurate description of the long-range part. The non-linear parameters as well as those of the weighting function (Eq. 4) were determined by the trial and error method, leading to the following values: $A_{0}=1.8 a_{0}^{-1}, R_{0}=20 a_{0}, \beta=6 a_{0}^{-1}, w_{\min }=0.001, w_{\max }=200$, $E_{0}=4000 \mathrm{~cm}^{-1}$, and $\alpha=1.73 \times 10^{-3} \mathrm{~cm}^{-1}$.

The quality of the fitting procedure was checked by means of the root-mean-square (RMS) error. The RMS error for negative interaction energies is $0.01 \mathrm{~cm}^{-1}$ while for energies in the range $0-5000 \mathrm{~cm}^{-1}$ it is $0.06 \mathrm{~cm}^{-1}$. For energies above $5000 \mathrm{~cm}^{-1}$ the RMS error increases more or less linearly with the energy. For instance, for energies in the range 5000-10000 $\mathrm{cm}^{-1}$ it is $59.6 \mathrm{~cm}^{-1}$ while the relative RMS error is only $0.66 \%$. These large errors are located at high energy in the short-range repulsive region of the PES and are then expected to have little to no consequences on the collisional dynamics at low temperatures which is the main purpose of the present PES.

\section{Features of the PES}

Four types of stationary points have been found on the PES of the $\mathrm{H}_{2}-\mathrm{C}_{3} \mathrm{~N}^{-}$system and are represented in Figure 2. These are the global minima MIN1, the secondary minima 


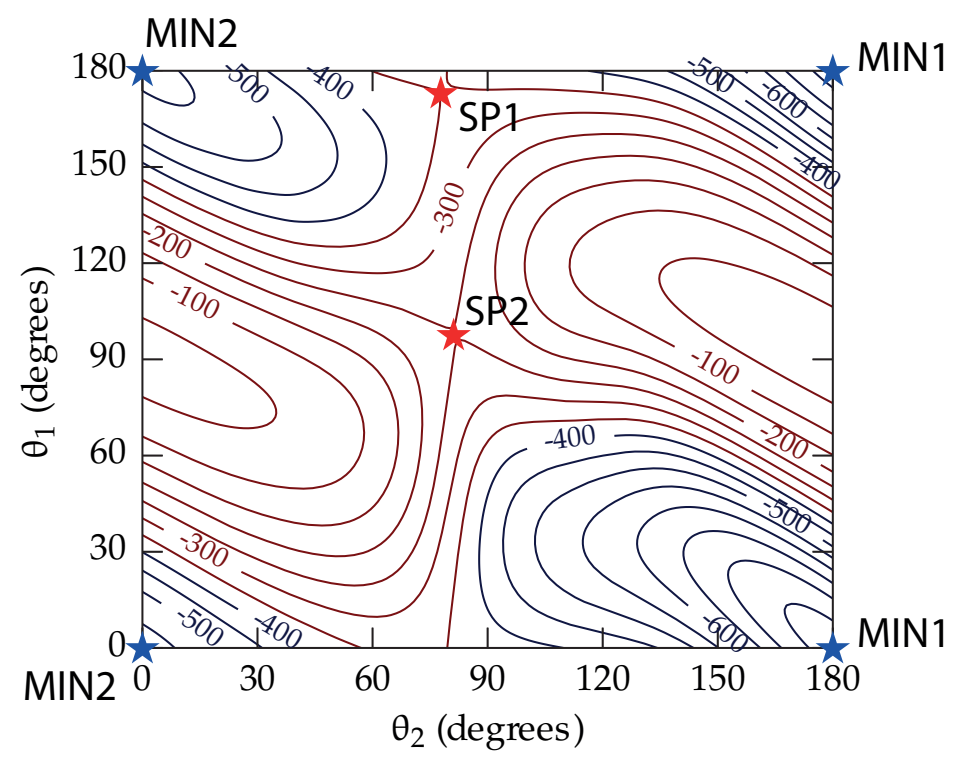

FIG. 2. (color online) 2D contour plot of the PES for $\phi=0^{\circ}$ and $R$ relaxed. Contour lines are equally spaced by $50 \mathrm{~cm}^{-1}$ and the energy zero corresponds to infinitely separated monomers.

MIN2, and the submerged saddle points SP1 and SP2. The geometries of the minima are collinear while those of the saddle points are planar, as shown in Table I. The $\mathrm{C}$ end and the $\mathrm{N}$ end of the $\mathrm{C}_{3} \mathrm{~N}^{-}$molecule are respectively pointing towards the $\mathrm{H}_{2}$ molecule in the MIN1 and MIN2 minima. Because of the exchange symmetry of the two $\mathrm{H}$ atoms, there are two equivalent stationary points for each of the two minima MIN1 and MIN2 and also for each of the saddle points SP1 and SP2. For the minima, the two equivalent stationary points are related by the symmetry operation $\theta_{1} \mapsto \theta_{1} \pm \pi$ while for the saddle points SP1 and SP2, the two equivalent stationary points are related by the symmetry operation $\theta_{1} \mapsto \pi-\theta_{1}$ and $\phi \mapsto \phi \pm \pi$. Both types of saddle points correspond to the transition states for the interconversion between a MIN1 and a MIN2 minima. In the case of the SP2 points, this interconversion is accompanied by a permutation of $\mathrm{H}$ atoms. There is no saddle point allowing a direct path from a MIN1 minimum to the other MIN1 minimum or from a MIN2 minimum to the other MIN2 minimum since the rotation of $\mathrm{H}_{2}$ is blocked by a large barrier of potential. All these important features of the PES result from its strong anisotropy with respect to the orientation of both monomers. 


\section{Long-range intermolecular forces}

In this section a physical analysis of the different contributions to the long-range interaction potential is presented in order to get a better understating of the nature of the interaction in the asymptotic region as well as to validate the extrapolation capabilities of the present PES. The long-range potential is as usual defined as the sum of the three electrostatic, induction and dispersion contributions,

$$
V=V_{\text {elec }}+V_{\text {ind }}+V_{\text {disp }}
$$

where each term is in turn expanded as in equation (3). Analytical forms of the leading expansion coefficients for these three different kind of contributions were then obtained by applying the approach of Buckingham ${ }^{25}$ and are shown in Table S1 (see supplementary material).

The values of the multipole moments and polarizabilities appearing in these expressions and which are used in the present work are given in Table II. They were calculated using the finite field method implemented in MOLPRO ${ }^{22}$ at the $\operatorname{CCSD}(\mathrm{T}) /$ aug-cc-pVQZ level. Also shown in Table II are the ionization energies of $\mathrm{H}_{2}$ and $\mathrm{C}_{3} \mathrm{~N}^{-}$, the later being considered to be equal to the electron affinity of its neutral counterpart.

Figure 3 shows a comparison between the long-range fitted coefficients of the PES (equation 5) and their values obtained by using the formulae of Table S1 (see supplementary material). A very good agreement is observed, although there are small differences at shorter distances which probably result from the neglect of higher order induction and dispersion terms which were excluded from our analysis. In the case of the dispersion contribution, the disagreement may also result from the inaccuracy of the London formula. In any case, the good agreement between both approaches demonstrates the good asymptotic physical behaviour of the present PES which is then suitable for describing cold molecular collisions. We furthermore conclude that the electrostatic charge-quadrupole interaction $\left(\propto R^{-3}\right)$, see Figure 3 gives the largest contribution to the asymptotic regions of the potential apart in a narrow $\theta_{1}$ interval where $\bar{P}_{2}^{0}\left(\cos \theta_{1}\right)$ is close to zero and where the charge-induced dipole interaction $\left(\propto R^{-4}\right)$ gives a larger contribution. 
TABLE II. Molecular properties of $\mathrm{H}_{2}$ and $\mathrm{C}_{3} \mathrm{~N}^{-}$needed in the calculation of long-range interactions. The multipole moments and polarizabilities values have been calculated with the origin at the center of mass. All values are in atomic units

\begin{tabular}{ccc}
\hline \hline Property & $\mathrm{H}_{2}$ & $\mathrm{C}_{3} \mathrm{~N}^{-}$ \\
\hline$\mu$ & 0 & 1.38 \\
$\Theta$ & 0.48 & -19.09 \\
$\alpha_{\|}$ & 6.72 & 83.93 \\
$\alpha_{\perp}$ & 4.74 & 38.1 \\
$A_{\|}$ & 0 & 115.97 \\
$A_{\perp}$ & 0 & 79.29 \\
$U$ & $0.64^{\mathrm{a}}$ & $0.16^{\mathrm{b}}$ \\
\hline \hline
\end{tabular}

${ }^{\text {a }}$ Reference 26

b Reference 15

\section{BOUND STATES}

\section{A. Method}

The rovibrational energy levels $E_{i}$ have been obtained by variationally solving the space-fixed rigid rotor Schrödinger equation:

$$
\begin{gathered}
{\left[\hat{H}\left(\mathbf{R}, \hat{\mathbf{r}}_{\mathbf{1}}, \hat{\mathbf{r}}_{\mathbf{2}}\right)-E_{i}\right] \Psi_{i}^{J M}\left(\mathbf{R}, \hat{\mathbf{r}}_{\mathbf{1}}, \hat{\mathbf{r}}_{\mathbf{2}}\right)=0} \\
\hat{H}\left(\mathbf{R}, \hat{\mathbf{r}_{\mathbf{1}}}, \hat{\mathbf{r}_{\mathbf{2}}}\right)=-\frac{\hbar^{2}}{2 \mu}\left[\frac{1}{R} \frac{\partial^{2}}{\partial R^{2}} R+\frac{\hat{l}^{2}}{R^{2}}\right]+\hat{H}_{1}\left(\hat{\mathbf{r}}_{\mathbf{1}}\right) \\
+\hat{H}_{2}\left(\hat{\mathbf{r}}_{\mathbf{2}}\right)+V\left(\mathbf{R}, \hat{\mathbf{r}}_{\mathbf{1}}, \hat{\mathbf{r}}_{\mathbf{2}}\right)
\end{gathered}
$$

where $\mathbf{R}$ stands for the intermolecular separation vector, $\hat{\mathbf{r}}_{1}$ and $\hat{\mathbf{r}}_{\mathbf{2}}$ for the angular coordinates of $\mathrm{H}_{2}$ and $\mathrm{C}_{3} \mathrm{~N}^{-}$, respectively, $\mu$ is the reduced mass, while $J$ and $M$ are the total angular momentum and its projection onto the space-fixed $z$-axis, respectively. The rigid rotor Hamiltonian $H_{i}$ of the linear molecule $i$ obeys the Schrödinger equation

$$
\hat{H}_{i}\left(\hat{\mathbf{r}}_{\mathbf{i}}\right) Y_{j_{i} m_{i}}\left(\hat{\mathbf{r}}_{\mathbf{i}}\right)=E_{j_{i}} Y_{j_{i} m_{i}}\left(\hat{\mathbf{r}}_{\mathbf{i}}\right)
$$




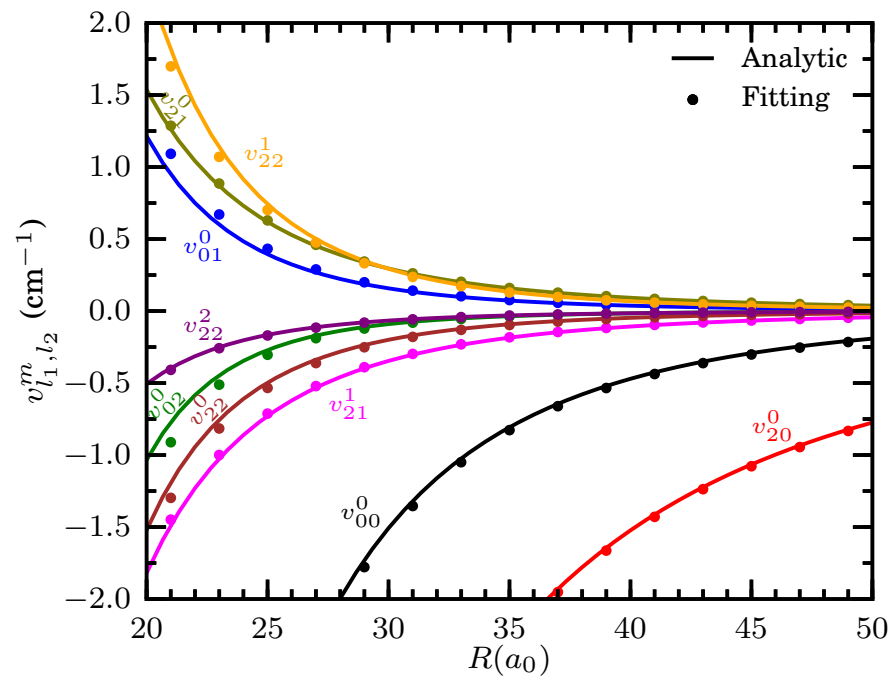

FIG. 3. (color online) Comparison between the two methods of calculation of the expansion coefficients $v_{l_{1}, l_{2}}^{m}(R)$ defined by equation (3). The coefficients are obtained from the fit of the ab initio data (solid circles) or by the formulae of Table S1 (solid lines), see supplementary material.

where $E_{j_{i}}=B_{i} j_{i}\left(j_{i}+1\right)$ and $Y_{j_{i} m_{i}}$ are spherical harmonic functions. The rotational constanst $B_{i}$ were set to their experimental values, namely $60.853 \mathrm{~cm}^{-1}$ for $\mathrm{H}_{2}{ }^{27}$ and $0.186 \mathrm{~cm}^{-1}$ for $\mathrm{C}_{3} \mathrm{~N}^{-23}$.

The rovibrational wavefunctions $\Psi_{i}^{J M}$ are as usual expanded over a product of radial and symmetry-adapted angular functions,

$$
\Psi_{i}^{J M}=\sum_{n k} c_{n k}^{i} R^{-1} G_{n}(R) \mathcal{Y}_{k}^{J M}\left(\hat{\mathbf{R}}, \hat{\mathbf{r}}_{1}, \hat{\mathbf{r}}_{\mathbf{2}}\right)
$$

where $k \equiv\left\{j_{1}, j_{2}, j_{12}, l\right\}$. The Sturmian functions $G_{i}(R)$ are used to obtain a discrete variable representation (DVR) of the radial part of these wave functions ${ }^{28}$ while the $\mathcal{Y}_{k}^{J M}\left(\hat{\mathbf{R}}, \hat{\mathbf{r}}_{\mathbf{1}}, \hat{\mathbf{r}}_{\mathbf{2}}\right)$ are the coupled spherical harmonics defined by

$$
\begin{aligned}
\mathcal{Y}_{k}^{J M}\left(\hat{\mathbf{R}}, \hat{\mathbf{r}}_{\mathbf{1}}, \hat{\mathbf{r}}_{\mathbf{2}}\right)= & \sum_{\text {all } m}\left\langle j_{1} m_{1} j_{2} m_{2} \mid j_{12} m_{12}\right\rangle\left\langle j_{12} m_{12} l m_{l} \mid J M\right\rangle \\
& \times Y_{j_{1} m_{1}}\left(\hat{\mathbf{r}}_{\mathbf{1}}\right) Y_{j_{2} m_{2}}\left(\hat{\mathbf{r}}_{\mathbf{2}}\right) Y_{l m_{l}}(\hat{\mathbf{R}})
\end{aligned}
$$

The symmetry-adapted angular functions $\mathcal{Y}_{k}^{J M}\left(\hat{\mathbf{R}}, \hat{\mathbf{r}}_{\mathbf{1}}, \hat{\mathbf{r}}_{\mathbf{2}}\right)$ span the irreducible representations (irreps) of the group $G_{4}$ (cf. supplementary material) which is the complete permutation inversion group ${ }^{29}$ of the $\mathrm{H}_{2}-\mathrm{C}_{3} \mathrm{~N}^{-}$system. Since the $G_{i}(R)$ radial functions belong to the totally symmetric representation, thus the rovibrational wavefunctions $\Psi_{i}^{J M}$ belong 
TABLE III. Classification of the symmetry-adapted angular basis set according to $G_{4}$ irreps as a function of the parity, even $(e)$ or odd $(o)$, of $j_{1}$ and $j_{2}+l$.

\begin{tabular}{ccc}
\hline \hline$j_{1}$ & $j_{2}+l$ & $\Gamma_{i}$ \\
\hline$e$ & $e$ & $A_{1}$ \\
$e$ & $o$ & $A_{2}$ \\
$o$ & $e$ & $B_{1}$ \\
$o$ & $o$ & $B_{2}$ \\
\hline \hline
\end{tabular}

to the same representation than the $\mathcal{Y}_{k}^{J M}\left(\hat{\mathbf{R}}, \hat{\mathbf{r}}_{\mathbf{1}}, \hat{\mathbf{r}}_{\mathbf{2}}\right)$ functions over which they are expanded (see Table III).

The final energies are obtained by a sequential diagonalization-truncation procedure ${ }^{30}$. First, for each point of the radial DVR, the angular dependent part of the Hamiltonian is diagonalized in the angular basis defined by equation (12), and then truncated by retaining only the eigenfunctions whose eigenvalues are smaller than $E_{c u t}=2000 \mathrm{~cm}^{-1}$. Secondly, the truncated angular basis set and the radial DVR are used to represent the full Hamiltonian which is then diagonalized. The convergence of the rovibrational energies with respect to the size of the basis set was then checked. By setting the convergence criterion at 0.001 $\mathrm{cm}^{-1}$, we found that for each symmetry, 30 and 4 rotational functions for $\mathrm{C}_{3} \mathrm{~N}^{-}$and $\mathrm{H}_{2}$, respectively, together with a 50 points radial DVR in the range 5-20 $a_{0}$ are required to reach convergence.

\section{B. Vibrational levels and wavefunctions}

The bound states energies for $J=0$ are shown in Tables IV and V. The rovibrational ground state is largely above the SP1 saddle points and slightly lower than the SP2 saddle points by $7.7 \mathrm{~cm}^{-1}$. All other bound states are above all the saddle points, therefore the rovibrational wavefunctions are expected to be delocalized over all minima.

Some physical insight on the nature of the vibrational wavefunctions can be extracted from a normal mode analysis based on the Jacobi coordinates defined in Figure 1. The coordinate $\phi$ is however not defined for all the minima which have a linear geometry. Therefore the harmonics frequencies and normal modes of the minima have been obtained using 
TABLE IV. Lowest $\mathrm{A}_{1}$ energy levels for $J=0, l_{v}=0$, and $v_{3}=0$, with their assigned vibrational quantum numbers $v_{1}$ and $v_{2}$. The wavefunctions can be localized in MIN1 minima or in MIN2 minima or delocalized over all minima. Energy is given in $\mathrm{cm}^{-1}$.

\begin{tabular}{|c|c|c|c|c|c|c|c|}
\hline \multirow[t]{2}{*}{ Level } & \multicolumn{2}{|c|}{ MIN1 } & \multicolumn{2}{|c|}{ MIN2 } & \multicolumn{2}{|c|}{ Global } & \multirow[t]{2}{*}{ Energy } \\
\hline & $v_{1}$ & $v_{2}$ & $v_{1}$ & $v_{2}$ & $v_{1}$ & $v_{2}$ & \\
\hline 0 & 0 & 0 & & & 0 & 0 & -257.22 \\
\hline 1 & 2 & 0 & & & 2 & 0 & -232.95 \\
\hline 2 & 4 & 0 & & & 4 & 0 & -216.34 \\
\hline 3 & 6 & 0 & & & 6 & 0 & -203.00 \\
\hline 4 & 8 & 0 & & & 8 & 0 & -189.93 \\
\hline 5 & 10 & 0 & 0 & 0 & 10 & 0 & -174.95 \\
\hline 6 & 10 & 0 & 0 & 0 & 12 & 0 & -174.76 \\
\hline 7 & 12 & 0 & 2 & 0 & 14 & 0 & -160.57 \\
\hline 8 & 12 & 0 & 2 & 0 & 16 & 0 & -157.08 \\
\hline 9 & 0 & 1 & & & 0 & 1 & -153.89 \\
\hline 10 & & & & & 18 & 0 & -146.80 \\
\hline 11 & & & & & 20 & 0 & -137.43 \\
\hline 12 & 2 & 1 & & & 2 & 1 & -134.08 \\
\hline 13 & & & & & 22 & 0 & -125.76 \\
\hline 14 & 4 & 1 & & & 4 & 1 & -120.79 \\
\hline 15 & & & & & 24 & 0 & -113.17 \\
\hline 16 & 6 & 1 & & & 6 & 1 & -109.80 \\
\hline 17 & 8 & 1 & & & 8 & 1 & -99.17 \\
\hline 18 & & & & & 26 & 0 & -98.14 \\
\hline 21 & 0 & 2 & & & 0 & 2 & -83.73 \\
\hline 22 & & & & & 28 & 0 & -80.74 \\
\hline
\end{tabular}

the Wilson's FG method ${ }^{31}$ restricted to the $3 \mathrm{D}$ space spanned by the coordinates $R, \theta_{1}$ and $\theta_{2}$. The results are given in Table VI. Since the PES is highly anharmonic, the FG harmonic frequencies are significantly different from the accurate fundamental frequencies. However, it is still possible to establish a correspondence between both types of frequencies and therefore to associate the normal mode displacements to the fundamental frequencies, except for the $\nu_{3}$ mode for which the harmonic frequency is larger than the dissociation energy. The high frequency of the $\nu_{3}$ mode can be understood if we observe the Figure 2 . Indeed, for $\theta_{2}=0^{\circ}$ or $\theta_{2}=180^{\circ}$, the motion along $\theta_{1}$ is blocked by a large potential barrier. 
TABLE $\mathrm{V}$. Lowest $\mathrm{B}_{2}$ energy levels for $J=0, l_{v}=0$, and $v_{3}=0$, with their assigned vibrational quantum numbers $v_{1}$ and $v_{2}$. The wavefunctions can be localized in MIN1 minima or in MIN2 minima or delocalized over all minima. Energy is given in $\mathrm{cm}^{-1}$.

\begin{tabular}{|c|c|c|c|c|c|c|c|}
\hline \multirow[t]{2}{*}{ Level } & \multicolumn{2}{|c|}{ MIN1 } & \multicolumn{2}{|c|}{ MIN2 } & \multicolumn{2}{|c|}{ Global } & \multirow[t]{2}{*}{ Energy } \\
\hline & $v_{1}$ & $v_{2}$ & $v_{1}$ & $v_{2}$ & $v_{1}$ & $v_{2}$ & \\
\hline 0 & 0 & 0 & & & 0 & 0 & -242.50 \\
\hline 1 & 2 & 0 & & & 2 & 0 & -214.84 \\
\hline 2 & 4 & 0 & & & 4 & 0 & -193.29 \\
\hline 3 & 6 & 0 & & & 6 & 0 & -174.67 \\
\hline 4 & 8 & 0 & & & 8 & 0 & -156.44 \\
\hline 5 & & & 0 & 0 & {$[10]$} & 0 & -141.71 \\
\hline 6 & 10 & 0 & & & {$[12]$} & 0 & -137.29 \\
\hline 7 & 0 & 1 & & & 0 & 1 & -125.74 \\
\hline 8 & & & 2 & 0 & [14] & 0 & -120.00 \\
\hline 9 & 12 & 0 & & & {$[16]$} & 0 & -116.38 \\
\hline 10 & & & 4 & 0 & [18] & 0 & -101.76 \\
\hline 11 & 2 & 1 & & & 2 & 1 & -101.45 \\
\hline 12 & 14 & 0 & 6 & 0 & 20 & 0 & -95.02 \\
\hline 13 & 14 & 0 & 6 & 0 & 22 & 0 & -84.47 \\
\hline 14 & 4 & 1 & & & 4 & 1 & -82.69 \\
\hline 15 & & & & & 24 & 0 & -71.89 \\
\hline 16 & 6 & 1 & & & 6 & 1 & -66.53 \\
\hline 17 & & & & & 26 & 0 & -59.89 \\
\hline 20 & 0 & 2 & & & 0 & 2 & -39.41 \\
\hline 22 & & & 0 & 1 & & 1 & -33.47 \\
\hline
\end{tabular}

We furthermore note that the bending modes $\nu_{1}$ and $\nu_{3}$ are doubly degenerate as a result of the linear geometry of the equilibrium structures.

The molecular symmetry group ${ }^{29}$ for linear molecules without a center of symmetry is called $C_{\infty v}(\mathrm{M})$ and is made of the two elements $E$ and $E^{*}$. The harmonic vibrational wavefunctions, which are localized in a single potential well, can be classified according to the $C_{\infty v}(\mathrm{M})$ group irreps as $\Sigma^{+}$or $\Sigma^{-}$if they are symmetric or antisymmetric with respect to the inversion operation $E^{*}$. The connection between the harmonic model and the full variational calculation can be deduced by invoking the permutation between the two identical $\mathrm{H}$ atoms. This operation transforms a minimum structure associated with $\theta_{1}=0^{\circ}$ 
TABLE VI. Frequencies and normal modes coordinates for the global (MIN1) and secondary (MIN2) minima of $\mathrm{H}_{2}-\mathrm{C}_{3} \mathrm{~N}^{-}$.

\begin{tabular}{ccccccc}
\hline \hline \multirow{2}{*}{ Mode } & & \multicolumn{2}{c}{ MIN1 } & & \multicolumn{2}{c}{ MIN2 } \\
\cline { 3 - 4 } \cline { 6 - 7 } & Displacement & Harmonic & Fundamental & & Harmonic & Fundamental \\
\hline$\nu_{1}$ & $\Delta \theta_{2}$ & 49.9 & 14.4 & & 44.8 & 8.8 \\
$\nu_{2}$ & $\Delta R$ & 196.8 & 103.3 & & 201.5 & - \\
$\nu_{3}$ & $\Delta \theta_{1}$ & 540.5 & - & & 420.7 & - \\
\hline \hline
\end{tabular}

into another equivalent one with $\theta_{1}=180^{\circ}$ or inversely. Therefore, the global wavefunctions can be expressed as linear combinations of two equivalent local wavefunctions:

$$
\begin{aligned}
& \Psi_{1}=\frac{1}{\sqrt{2}}\left(\varphi_{0^{\circ}}+\varphi_{180^{\circ}}\right) \\
& \Psi_{2}=\frac{1}{\sqrt{2}}\left(\varphi_{0^{\circ}}-\varphi_{180^{\circ}}\right)
\end{aligned}
$$

These combinations lead to the following correspondence between the irreps of the $C_{\infty v}(\mathrm{M})$ and $G_{4}$ groups:

$$
\begin{aligned}
& 2 \Sigma^{+}=A_{1} \oplus B_{2} \\
& 2 \Sigma^{-}=A_{2} \oplus B_{1}
\end{aligned}
$$

In our case, the normal modes coordinates and the harmonic vibrational wavefunctions are invariant with respect to inversion. Thus they belong to the $\Sigma^{+}$irrep. Hence, for $J=0$, we can obtain only global vibrational wavefunctions belonging to the $A_{1}$ and $B_{2}$ irreps. In the harmonic approximation, vibrational energies levels belonging to $A_{1}$ are degenerate with those of the $B_{2}$. In the variational approach, which is based on a realistic PES, these levels are no longer degenerate since they are coupled by the PES. For instance, the energy splitting is about $15 \mathrm{~cm}^{-1}$ between the ground state of the symmetry $A_{1}$ (denoted by $A_{1} .0$ ) and the corresponding ground state of the symmetry $B_{2}$ (denoted by $B_{2} .0$ ). Hereinafter, the levels are denoted by the symbol of the irrep followed by the number of the state in the series of states belonging to the same irrep, ordered by increasing energy.

Contour plots of selected wavefunctions for $J=0$ are shown in Figure 4. Let us remind that the two equivalent global minima MIN1 are located at $\theta_{2}=180^{\circ}$ while the two equiv- 

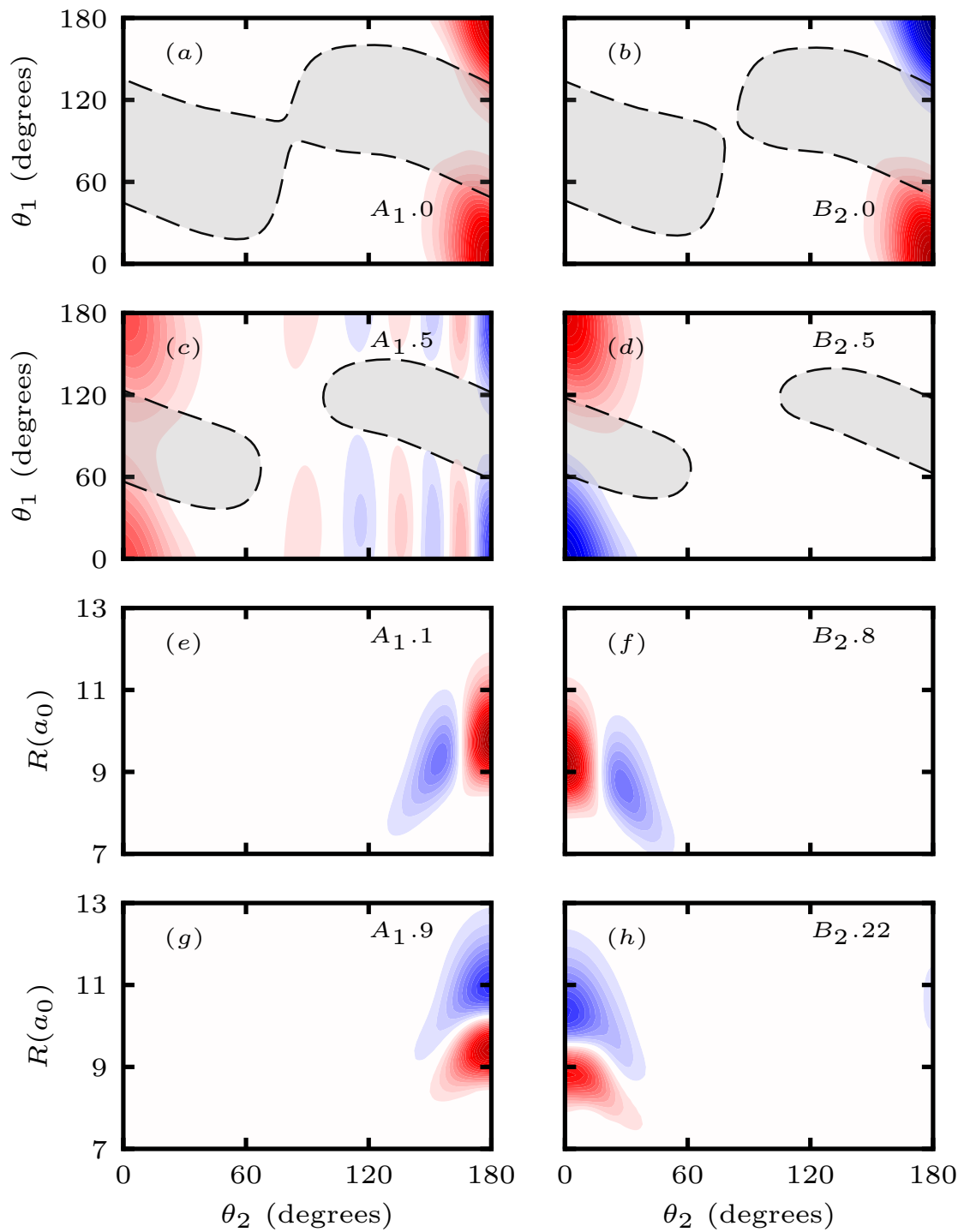

FIG. 4. (color online) 2D contour plots for selected $J=0$ rovibrational wavefunctions. Red contours correspond to positive amplitude and blue contours to negative amplitude. a-d) $R$ is relaxed and $\phi=0^{\circ}$; The shaded areas show the classically forbidden regions at the energy of the vibrational level. e-h) $\theta_{1}=0^{\circ}$ and $\phi=0^{\circ}$.

alent secondary minima MIN2 are at $\theta_{2}=0^{\circ}$. The panels $(a)$ and $(b)$ of Figure 4 show the ground state of each symmetry, $A_{1}$ and $B_{2}$. Although their energies are above the saddle points, these wavefunctions are noticeably localized in the MIN1 region. In the panels $(c)$ and $(d)$ are shown the wavefunctions which can be described as the ground states of the secondary minima since they are the lowest levels clearly localized in the MIN2 region. These 

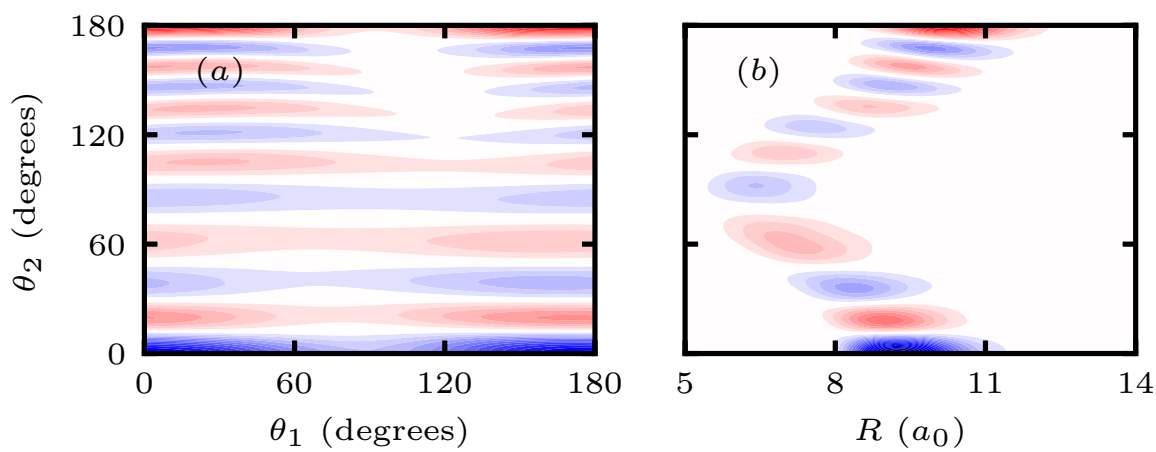

FIG. 5. (color online) $2 \mathrm{D}$ contour plots of the $A_{1} .13$ rovibrational wavefunction for $J=0$. Red contours correspond to positive amplitude and blue contours to negative amplitude.

levels are labeled as $A_{1} .5$ and $B_{2} .5$. Again, although their energies are significantly above the MIN1 $\leftrightarrow$ MIN2 interconversion barriers, these wavefunctions remain mostly localized in the MIN2 region. One can however observe in Figure $4(c)$ a mixing with a $\nu_{1}$ overtone localized in the global minimum MIN1. Such anharmonic resonances can be seen in many $A_{1}$ rovibrational levels, while in contrast they have a low occurrence for the $B_{2}$ states. Figure $4(c)$ shows also that the $A_{1} .5$ wavefunction span the whole range of variation of $\theta_{1}$, thus revealing a strong tunneling effect between the two equivalent MIN2 minima, even though the potential barrier is higher by about $125 \mathrm{~cm}^{-1}$ than the vibrational level $A_{1} .5$.

The first excited levels localized around the global minima MIN1, labeled by $A_{1} .1$ and $B_{2} .1$, correspond to a vibrational excitation in the mode $\nu_{1}$. The corresponding levels for the secondary minima MIN2 are $A_{1} .7$ and $B_{2} .8$. The levels $A_{1} .1$ and $B_{2} .8$ are shown in Figure 4 $(e)$ and $(f)$, respectively. Because of the small value of the $\nu_{1}$ frequency for both MIN1 and MIN2 minima, many of the rovibrational levels are either pure or mixed $\nu_{1}$ overtones.

The fundamental stretching mode is identified in levels $A_{1} .9$ and $B_{2} .7$ for the global minima MIN1, while for the secondary minima MIN2, it appears in levels $A_{1} .20$ and $B_{2} .22$. The levels $A_{1} .9$ and $B_{2} .22$ are shown in Figure $4(g)$ and $(h)$, respectively. Overtones and combination tones of the stretching mode $\nu_{2}$ also occur although in a minor extent because of the high frequency of this mode.

The list of the lowest levels for which it is possible to assign vibrational quantum numbers is shown in Tables IV and V. Only levels with $J=0$ are shown, and therefore the vibrational angular momentum $l_{v}$ associated to each doubly degenerate bending motion is also equal to zero. This imply an equal number of quanta in both states of each doubly degenerate 
bending motions. Only the $v_{1}$ and $v_{2}$ quantum numbers are reported in Tables IV and V since no excited state was found for the $\nu_{3}$ bending motion. As a matter of fact, this mode has a very large harmonic vibrational frequency, much larger than the dissociation energy.

Four cases appear when we examine the nodal pattern of the wavefunctions with respect to the rotation of $\mathrm{C}_{3} \mathrm{~N}^{-}$, i.e. the coordinate $\theta_{2}$. In case 1 or 2 , the wavefunction is localized in MIN1 or in MIN2 minima respectively, as shown in panels $(a)$ and $(d)$ of Figure 4. In case 3, the wavefunction is a combination of two local wavefunctions in MIN1 and MIN2 minima as for example shown in panel $(c)$ of Figure 4. In case 4, the wavefunction is spanning the whole range of variation of $\theta_{2}$, as shown in Figure 5. In order to distinguish between these cases, three different lists of quantum numbers $v_{1}$ and $v_{2}$ are reported in Tables IV and V. When the vibrational wavefunction is clearly localized in the MIN1 minima, or in the MIN2 minima, or in both, then we assign quantum numbers to these local wavefunctions. The third list of quantum numbers is associated to the global wavefunction, which can be a wavefunction belonging to any of the four cases.

Table IV shows that levels 0-4 are localized only in MIN1 while levels 5-8 are localized in MIN1 and MIN2 and are associated in symmetric or antisymmetric combinations, the antisymmetric combination adding one nodal plane in both degenerate states of the bending motion $\nu_{1}$. Some higher levels, such as levels 10,11 and 13 for example, are fully delocalized (see Figure 5) and therefore only global quantum numbers can be assigned to these levels. Whereas global quantum numbers can be assigned to all the $A_{1}$ levels listed in Table IV, such an assignation does not appear to be possible for all the $B_{2}$ levels listed in Table $\mathrm{V}$ as the wavefunctions of these levels which are localized in the MIN2 minima are not mixed with those localized in the MIN1 minima or vice versa. This is the case of the $B_{2} .5, B_{2} .6, B_{2} .8$, $B_{2} .9$, and $B_{2} .10$ levels. However, if we assume that the states are mixed, for example $B_{2} .5$ and $B_{2} .6$, then symmetric and antisymmetric combinations can be done and a hypothetical $v_{1}$ global quantum number can be defined. In Table $\mathrm{V}$, the hypothetical quantum numbers are marked with square braket.

Another important feature of this system is that a significant fraction of the vibrational wavefunctions are localized in small regions of the coordinate space although they could be delocalized over large regions since their energy is well above the interconversion barriers. This can be understood by analyzing the distribution of the vibrational energy among the mode of motions. The vibrational stretching motion frequency $\nu_{2}$ is relatively large, around 
$103 \mathrm{~cm}^{-1}$, while the frequency $\nu_{3}$ which corresponds to the hindered rotation of $\mathrm{H}_{2}$, is even larger. Therefore, these two modes of motion withhold a large part of the vibrational energy, but they are not efficient for triggering the MIN1 $\leftrightarrow$ MIN2 interconversion since this needs a rotation of $\mathrm{C}_{3} \mathrm{~N}^{-}$. It is mainly the bending mode $\nu_{1}$ which triggers the interconversion. Only the levels with enough energy in this last mode of motion are delocalized over both minima, MIN1 and MIN2, such as, for instance, the level $A_{1} .5$ shown in Figure $4(c)$.

a. Vibrational frequencies The $\nu_{2}$ fundamental frequency associated with the MIN1 minima can be extracted from Table IV, but not the $\nu_{1}$ fundamental frequency since the level $v_{1}=1$ is not allowed for $J=0$. Indeed, $v_{1}=1$ implies $l_{v}= \pm 1$, which in turn implies

$J \geq 1$. We have thus computed the $J=1$ bound states and obtained the $\nu_{1}$ fundamental frequency for both MIN1 and MIN2 minima. These results are shown in Table VI along with the harmonic frequencies. The differences between both sets of data reveal the strong anharmonicities of the vibrational motions. A rough estimate of the $\nu_{3}$ fundamental frequency can also be obtained from the equation $\nu_{3}=\mathrm{ZPE}-\nu_{1}-\nu_{2} / 2$. This gives a frequency value of $446 \mathrm{~cm}^{-1}$, which is much larger than the dissociation energy, $257 \mathrm{~cm}^{-1}$.

b. Rotational Constants Since the MIN1 and MIN2 equilibrium structures of $\mathrm{H}_{2}-\mathrm{C}_{3} \mathrm{~N}^{-}$ are linear, there are only two equal rotational constants for each structure which can be calculated either by diagonalizing the inertia tensor or from the evaluation of the energy differences between the $J=0$ and $J=1$ ground levels. In the first case, we obtain 0.111 $\mathrm{cm}^{-1}$ and $0.115 \mathrm{~cm}^{-1}$ for the MIN1 and MIN2 equilibrium structures, respectively while in the second case, we obtain $0.165 \mathrm{~cm}^{-1}$ and $0.265 \mathrm{~cm}^{-1}$. However for the MIN2 structure, the energy difference between the $J=0$ and $J=1$ levels involve mixed states, i.e. states which are not fully localized in the MIN2 potential wells, as shown in panel (c) of Figure 4.

\section{Para and ortho states}

Since the nuclear spin of hydrogen atom is $I_{\mathrm{H}}=\frac{1}{2}$, the wavefunction of $\mathrm{H}_{2}$ must be antisymmetric under the exchange of the two nuclei, as required by the Pauli's principle. Therefore, the total wavefunction of $\mathrm{H}_{2}-\mathrm{C}_{3} \mathrm{~N}^{-}$which is given by the product $\Psi_{\text {elec }} \times \Psi_{\text {rovib }} \times \Psi_{\text {spin }}$ must belong to the irreps $B_{1}$ or $B_{2}$ of the group $G_{4}$. The electronic ground state wavefunction $\Psi_{\text {elec }}$ belongs to the totally symmetric representation. The rovibrational wavefunctions $\Psi_{\text {rovib }}$ can belong to any of the $G_{4}$ irreps for $J \geq 1$ and only to $A_{1}$ and $B_{2}$ for $J=0$. We can 
obtain the symmetry representation of the spin wavefunctions $\Psi_{\text {spin }}$ by considering the most abundant isotopes ${ }^{1} \mathrm{H},{ }^{12} \mathrm{C},{ }^{14} \mathrm{~N}$ whose nuclear spin are $I_{\mathrm{H}}=\frac{1}{2}, I_{\mathrm{C}}=0, I_{\mathrm{N}}=1$. Hence, the $\mathrm{C}_{3} \mathrm{~N}^{-}$nuclear spin is $I=1$ while the nuclear spin of $\mathrm{H}_{2}$ can be $I=0$ for $\left(\right.$ para- $\left.\mathrm{H}_{2}\right)$ or $I=1$ for $\left(\right.$ ortho- $\left.\mathrm{H}_{2}\right)$. The coupling of the singlet spin state of $\mathrm{H}_{2}$, which is antisymmetric under the exchange of nucleus, with the triplet spin state of $\mathrm{C}_{3} \mathrm{~N}^{-}$gives an antisymmetric triplet spin state for $\mathrm{H}_{2}-\mathrm{C}_{3} \mathrm{~N}^{-}$. On the other side, the coupling of the triplet spin state of $\mathrm{H}_{2}$, which is symmetric under the exchange of nucleus, with the triplet spin state of $\mathrm{C}_{3} \mathrm{~N}^{-}$gives a singlet, a triplet, and a quintet spin states for $\mathrm{H}_{2}-\mathrm{C}_{3} \mathrm{~N}^{-}$which are all symmetric under $\mathrm{H}$ exchange. Using now the table of characters of the $G_{4}$ group and noting that all spin functions are symmetric under the inversion operator $E^{*}$, we see that the symmetric (under exchange) spin functions of $\mathrm{H}_{2}-\mathrm{C}_{3} \mathrm{~N}^{-}$belong to the $A_{1}$ irrep, while the antisymmetric spin functions belong to the $B_{2}$ irrep.

Therefore, the spin states with symmetry $A_{1}(I=0,1,2)$ are combined with the $B_{1}$ and $B_{2}$ rovibrational states to give the ortho states of $\mathrm{H}_{2}-\mathrm{C}_{3} \mathrm{~N}^{-}$while the $B_{2}$ spin states $(I=1)$ are combined with the $A_{1}$ and $A_{2}$ rovibrational states to give the para states. The name ortho(para) is given to the states of the complex that asymptotically correlate with the dissociation limit ortho- $\mathrm{H}_{2}\left(\right.$ para $\left.-\mathrm{H}_{2}\right)+\mathrm{C}_{3} \mathrm{~N}^{-}$. Since transitions between ortho and

para states are forbidden one can consider them as two different species of the $\mathrm{H}_{2}-\mathrm{C}_{3} \mathrm{~N}^{-}$ complex. The rovibrational ground state of the para form is more stable than the ortho one by approximately $15 \mathrm{~cm}^{-1}$.

\section{CONCLUSION}

A new 4D PES which accounts for the interaction between $\mathrm{H}_{2}$ and the rigid $\mathrm{C}_{3} \mathrm{~N}^{-}$has been presented. This PES has been designed for the study of collisional dynamics at low temperatures. We carefully checked the accuracy of the PES taking a peculiar care of the long-range interactions which accurately describe the asymptotic limit of dissociation. Two different linear equilibrium structures have been found, one with the $\mathrm{C}$ end of $\mathrm{C}_{3} \mathrm{~N}^{-}$ pointing towards $\mathrm{H}_{2}$ which is the global minimum, and the other one with the $\mathrm{N}$ end of $\mathrm{C}_{3} \mathrm{~N}^{-}$pointing towards $\mathrm{H}_{2}$ which is the secondary minimum. Since the PES is symmetric under the exchange of the two $\mathrm{H}$ atoms, there is a total a four minima in the PES.

The rovibrational dynamics of the $\mathrm{H}_{2}-\mathrm{C}_{3} \mathrm{~N}^{-}$van der Waals complex has been investi- 
gated, considering $\mathrm{H}_{2}$ and $\mathrm{C}_{3} \mathrm{~N}^{-}$as linear rigid rotors. The examination of the rovibrational wavefunctions has revealed some quantum features such as unexpected wavefunction localization, tunneling effect and anharmonic resonances. The $\mathrm{H}_{2}-\mathrm{C}_{3} \mathrm{~N}^{-}$system exhibits two doubly degenerate bending modes. One corresponds mainly to the hindered rotation of $\mathrm{C}_{3} \mathrm{~N}^{-}$and the other mainly to the hindered rotation of $\mathrm{H}_{2}$. The vibrational dynamics of $\mathrm{H}_{2}-\mathrm{C}_{3} \mathrm{~N}^{-}$is principally controlled by the considerable difference between the two bending frequencies. This difference results on one side from the large difference in mass between $\mathrm{H}_{2}$ and $\mathrm{C}_{3} \mathrm{~N}^{-}$and on the other side from the potential which is soft for the rotation of $\mathrm{C}_{3} \mathrm{~N}^{-}$and stiff for the rotation of $\mathrm{H}_{2}$. The bending/rotation of $\mathrm{C}_{3} \mathrm{~N}^{-}$, which triggers the interconversion MIN1 $\leftrightarrow$ MIN2, is largely allowed since the saddle points energies are below those of almost all vibrational levels. But since much of the available vibrational energy is withholded by the bending/rotation motion of $\mathrm{H}_{2}$ and also by the stretching motion, the wavefunctions remain localized in the MIN1 minima or in the MIN2 minima. In contrast, the bending/rotation of $\mathrm{H}_{2}$, which should allow transforming one MIN1 minimum into the other or one MIN2 minimum into the other, is blocked by a high potential barrier. But since $\mathrm{H}_{2}$ is light, a significant tunneling effect is observed which causes a lifting of the degeneracy between symmetric and antisymmetric wavefunctions localized in equivalent potential wells by about $15 \mathrm{~cm}^{-1}$.

Fundamental frequencies and rotational constants have been determined for both type of minima of the complex. For the MIN1 and MIN2 equilibrium structures, the fundamental frequencies of the mode of motion which corresponds mainly to the hindered rotation of $\mathrm{C}_{3} \mathrm{~N}^{-}$are $14.4 \mathrm{~cm}^{-1}$ and $8.8 \mathrm{~cm}^{-1}$, respectively. For the MIN1 equilibrium structure, the fundamental frequency of the intermonomer stretching mode of motion is $103.3 \mathrm{~cm}^{-1}$. The rotational constants, calculated by the difference of energy between rovibrational levels, are $0.165 \mathrm{~cm}^{-1}$ and $0.265 \mathrm{~cm}^{-1}$ for the MIN1 and MIN2 equilibrium structures, respectively.

\section{SUPPLEMENTARY MATERIAL}

See supplementary material for a Fortran subroutine that computes the $\mathrm{H}_{2}-\mathrm{C}_{3} \mathrm{~N}^{-} \mathrm{PES}$ and also for four tables: the long-range interaction formulae, the character table of the $G_{4}$ group and the complete lists of the vibrational energy levels supported by the $\mathrm{H}_{2}-\mathrm{C}_{3} \mathrm{~N}^{-}$ PES for $J=0$ and $J=1$. 


\section{ACKNOWLEDGMENTS}

This research has been supported by the Agence Nationale de la Recherche (Project ANR-AnionCosChem) and the French embassy in Cuba. Computer time for this study was

provided by the Mésocentre de Calcul Intensif Aquitain which is the computing facilities of Université de Bordeaux et Université de Pau.

\section{REFERENCES}

${ }^{1}$ A. Dalgarno and R. A. McCray, Astrophys. J. 181, 95 (1973).

${ }^{2}$ E. Herbst, Nature 289, 656 (1981).

${ }^{3}$ P. Sarre, J. Chim. Phys. 77, 769 (1980).

${ }^{4}$ R. P. A. Bettens and E. Herbst, Astrophys. J. 468, 686 (1996).

${ }^{5}$ T. J. Millar, E. Herbst, and R. P. A. Bettens, Mon. Not. R. Astron. Soc. 316, 195 (2000).

${ }^{6}$ R. Terzieva and E. Herbst, Int. J. Mass Spectrom. 201, 135 (2000).

${ }^{7}$ Y. Morisawa, H. Hoshina, Y. Kato, Z. Simizu, S. Kuma, N. Sogoshi, M. Fushitani, S. Watanabe, Y. Miyamoto, T. Momose, Y. Kasai, and K. Kawaguchi, Publ. Astron. Soc. Jap. 57, 325 (2005).

${ }^{8}$ M. C. McCarthy, C. A. Gottlieb, H. Gupta, and P. Thaddeus, Astrophys. J. Lett. 652, L141 (2006).

${ }^{9}$ M. Agúndez, J. Cernicharo, M. Guélin, M. Gerin, M. C. McCarthy, and P. Thaddeus, Astron. Astrophys. 478, L19 (2008).

${ }^{10}$ M. Agúndez, J. Cernicharo, M. Guélin, C. Kahane, E. Roueff, J. Kłos, F. J. Aoiz, F. Lique, N. Marcelino, J. R. Goicoechea, M. G. García, C. A. Gottlieb, M. C. McCarthy, and P. Thaddeus, Astron. Astrophys. 517, L2 (2010).

${ }^{11}$ S. Brünken, H. Gupta, C. A. Gottlieb, M. C. McCarthy, and P. Thaddeus, Astrophys. J. Lett. 664, L43 (2007).

${ }^{12}$ J. Cernicharo, M. Guélin, M. Agúndez, K. Kawaguchi, M. McCarthy, and P. Thaddeus, Astron. Astrophys. 467, L37 (2007).

${ }^{13}$ J. Cernicharo, M. Guélin, M. Agúndez, M. C. McCarthy, and P. Thaddeus, Astrophys. J. Lett. 688, L83 (2008). 
${ }^{14}$ P. Thaddeus, C. A. Gottlieb, H. Gupta, S. Brünken, M. C. McCarthy, M. Agúndez, M. Guélin, and J. Cernicharo, Astrophys. J. 677, 1132 (2008).

${ }^{15}$ T. A. Yen, E. Garand, A. T. Shreve, and D. M. Neumark, J. Phys. Chem. A 114, 3215 (2010).

${ }^{16}$ T. J. Millar, C. Walsh, and T. A. Field, Chem. Rev. 117, 1765 (2017), pMID: 28112897.

${ }^{17}$ P. Halvick, T. Stoecklin, F. Lique, and M. Hochlaf, J. Chem. Phys. 135, 044312 (2011).

${ }^{18}$ C. Orek, J. Kłos, F. Lique, and N. Bulut, J. Chem. Phys. 144, 204303 (2016).

${ }^{19}$ R. A. Kendall, T. H. Dunning, and R. J. Harrison, J. Chem. Phys. 96, 6796 (1992).

${ }^{20}$ F. Weigend, A. Köhn, and C. Hättig, J. Chem. Phys. 116, 3175 (2002).

${ }^{21}$ F. Weigend, Phys. Chem. Chem. Phys. 4, 4285 (2002).

${ }^{22}$ H.-J. Werner, P. J. Knowles, G. Knizia, F. R. Manby, M. Schütz, P. Celani, T. Korona, R. Lindh, A. Mitrushenkov, G. Rauhut, K. R. Shamasundar, T. B. Adler, R. D. Amos, A. Bernhardsson, A. Berning, D. L. Cooper, M. J. O. Deegan, A. J. Dobbyn, F. Eckert, E. Goll, C. Hampel, A. Hesselmann, G. Hetzer, T. Hrenar, G. Jansen, C. Köppl, Y. Liu, A. W. Lloyd, R. A. Mata, A. J. May, S. J. McNicholas, W. Meyer, M. E. Mura, A. Nicklass, D. P. O’Neill, P. Palmieri, D. Peng, K. Pflüger, R. Pitzer, M. Reiher, T. Shiozaki, H. Stoll, A. J. Stone, R. Tarroni, T. Thorsteinsson, and M. Wang, "Molpro, version 2012.1, a package of ab initio programs," (2012), see http://www.molpro.net.

${ }^{23}$ R. Kołos, M. Gronowski, and P. Botschwina, J. Chem. Phys. 128, 154305 (2008).

${ }^{24}$ S. Boys and F. Bernardi, Mol. Phys. 19, 553 (1970).

${ }^{25}$ A. D. Buckingham, in Intermolecular Interactions: From Diatomics to Biopolymers (Perspectives in Quantum Chemistry $\&$ Biochemistry), edited by B. Pullman (John Wiley \& Sons, Inc., New York, 1978) pp. 1-67.

${ }^{26}$ D. Shiner, J. M. Gilligan, B. M. Cook, and W. Lichten, Phys. Rev. A 47, 4042 (1993).

${ }^{27}$ G. Herzberg and L. L. Howe, Can. J. Phys. 37, 636 (1959).

${ }^{28}$ M. Lara-Moreno, T. Stoecklin, and P. Halvick, J. Chem. Phys. 146, 224310 (2017).

${ }^{29}$ P. R. Bunker and P. Jensen, Molecular Symmetry and Spectroscopy, 2nd ed. (NRC Research Press, 1998).

${ }^{30}$ J. C. Light and Z. Bačić, J. Chem. Phys. 87, 4008 (1987).

${ }^{31}$ E. Wilson, J. Decius, and P. Cross, Molecular Vibrations: The Theory of Infrared and Raman Vibrational Spectra, Dover Books on Chemistry (Dover Publications, 2012). 